\title{
An Open Label Study investigating the Efficacy of Hypericum perforatum Special Extract (ZE117), Nicotine Patches and Combination (ZE117)/ Nicotine Patches for Smoking Cessation
}

\author{
Con Stough ${ }^{1,2 *}$, Andrew Scholey ${ }^{1,2}$, Christina Kure ${ }^{1,2}$, Joanne Tarasuik $^{1,2}$, Marni Kras ${ }^{1,2}$, Andrea Zangara ${ }^{1}$
}

${ }^{1}$ Brain Sciences Institute, Swinburne University of Technology, Melbourne, Victoria, Australia

${ }^{2}$ Swinburne Centre for Human Psychopharmacology, Swinburne University of Technology, Melbourne, Australia

\begin{abstract}
Introduction: Nicotine addiction has become one of the largest international health problems, increasing the risk of cardiovascular disease, cancers, and respiratory diseases. There are several treatments available for smoking cessation with the most common transdermal nicotine replacement and anti-depressants. However current treatments are only mildly efficacious and have side effects which lead to a decrease in their effectiveness. Additional treatments for smoking cessation with lower side effects are required.

Methods: We examined the efficacy of Remotiv ${ }^{\circledR}$ (Hypericum perforatum Special Extract - ZE117) compared to Nicabate ${ }^{\circledR}$ CQ Nicotine Replacement Therapy (NRT) and combination ZE117/NRT for smoking cessation over a treatment time of 10 weeks. Given the different pharmacological profiles of Ze117 and NRT it was expected that a combination treatment would be most efficacious for smoking cessation than the other two treatments alone. Sixty smokers aged between 18 and 60 years were enrolled in the study. Smoking status was assessed using CO levels measured by the Bedfont Smokerlyzer (primary outcome measure). Secondary outcome measures of anxiety, DSMIV nicotine dependence and Fagerström craving and symptoms were also recorded.

Results: A significant main effect of reduction in the number of smokers over the course of the study was observed although there were no significant differences between any of the treatment groups on this variable after 14 weeks.

Conclusions: These results indicate that the three treatment options are equally efficacious for smoking cessation. Interestingly the Ze117 treatment significantly reduced the amount of craving over the treatment duration of the study compared to the other two treatments indicating a possible mechanism by which Ze117 may assist in smoking cessation.
\end{abstract}

Keywords: Hypericum perforatum; Nicotine replacement therapy; Smoking cessation; Ze 117; Nicabate; Remotiv

\section{Introduction}

Nicotine addiction has become one of the largest international health problems, increasing the risk of life threatening diseases. It is estimated that smoking causes an alarming $22 \%$ of cardiovascular disease, $30 \%$ of all cancers, $70-90 \%$ of lung cancers and $56-80 \%$ of respiratory diseases [1]. With $21 \%$ of the Australian adult population classed as regular smokers, cigarette smoking has been reported to be responsible for 19,000 Australian deaths each year [2]. Smokingrelated illnesses contribute significantly to healthcare costs and pose a considerable burden on the healthcare system and are estimated to cost the Australian community $\$ 21$ billion dollars annually [3]. Even for those who choose to quit smoking, cessation may be difficult and is often unsuccessful. Community quit rates for smokers attempting to quit without pharmacological treatment or supportive counselling have been reported as $17 \%$ [4], which should be seen as unacceptably low given the negative health consequences of smoking.

Drugs of addiction exert influence over the brain reward pathway [5]. It is thought that dopaminergic stimulation of the nucleus accumbens reinforces use of the drug [6]. Addictive drugs may either directly influence the action of the neurotransmitter dopamine, or alter the activity of other neurotransmitters that exert a modulatory influence over the mesolimbic dopaminergic pathway [5]. Gamma aminobutyric acid (GABA), opioid, serotonergic, cholinergic and noradrenergic neurotransmitter pathways have all been shown to interact at various points along the mesolimbic dopaminergic pathway and to modulate its activity [7].

As one of the most addictive drugs of abuse, nicotine exerts direct influence on nicotinic acetylcholine $(\mathrm{ACh})$ receptors located on the cell bodies of dopamine neurons within the ventral tegmental area (VTA) [8]. Activation of these nicotinic ACh receptors increases the action of dopamine neurons, resulting in stimulation of the nucleus accumbens $[9,10]$. Additionally, nicotinic receptors located on other neurotransmitter inputs to the VTA may further increase dopamine release by removing the inhibitory influence of other neurotransmitters (i.e. decrease GABA and increase Glutamate [7,8].

The biology of nicotine addiction has also been found to be linked to changes in serotonergic transmission associated with chronic use [11]. Acute smoking has been found to increase serotonin release,

*Corresponding author: Con Stough, Swinburne University of Technology, PO Box 218 Hawthorn, Victoria, 3122, Australia, Tel: +61 39214 8167; Fax: +61 39214 4397; E-mail: cstough@swin.edu.au

Received September 04, 2013; Accepted December 09, 2013; Published December 11, 2013

Citation: Stough C, Scholey A, Kure C, Tarasuik J, Kras M, et al. (2013) An Open Label Study investigating the Efficacy of Hypericum perforatum Special Extract (ZE117), Nicotine Patches and Combination (ZE117)/Nicotine Patches for Smoking Cessation. Altern Integ Med 2: 147. doi:10.4172/2327-5162.1000147

Copyright: (C) 2013 Stough C, et al. This is an open-access article distributed under the terms of the Creative Commons Attribution License, which permits unrestricted use, distribution, and reproduction in any medium, provided the original author and source are credited. 
while chronic smoking decreasing the concentration and synthesis of brain serotonin [12]. An increase in serotonin transporter density in the prefrontal cortex has also been found to be associated with chronic nicotine administration $[13,14]$. During nicotine withdrawal there appears to be an increased sensitivity of serotonin neurons in the dorsal raphe nucleus [15].

Current pharmacological methods for treating smoking addiction in Australia that are approved by the government regulator include Nicotine Replacement Therapy (NRT) and Bupropion [16]. In the USA, the Food and Drug Administration (FDA) have approved a number of Nicotine Replacement Therapies including nicotine patches, gum, lozenges, sprays and inhalers [17]. There have also been two other pharmacological treatments approved by the FDA: Varenicline and Buproprion [18]. Randomised trials have found Buproprion to be effective in assisting cessation of smoking. Hurt et al., [19] reported the seven week quit rate for Bupropion to be significantly greater than the placebo quit rate of $19 \%$. While Bupropion has been shown to be effective in the management of smoking cessation, there are significant adverse effects associated with its use including seizures, insomnia, dizziness, headache and skin reactions [16]. Although not substantiated by research, there have been anecdotal reports of sudden death associated with Bupropion Two randomised controlled trials have reported that 12 weeks treatment with Varenicline to be more effective than Bupropion in the maintenance of continuous abstinence rates during weeks 9 to 24 after quit date [20-22]. Quit rates associated with Varenicline were reported to be $44 \%$ and $43.9 \%$, while quit rates associated with Bupropion were $29.5 \%$ and $29.8 \%$, and quit rates for placebo were $14 \%$ and $17.7 \%$ [20-22]. A number of adverse side effects associated with Varenicline use were reported in these studies, including nausea, headache, insomnia and abnormal dreams [20-22]. Discontinuation of treatment due to adverse events occurred in $8.6 \%$ and $10.5 \%$ of recipients in the Varenicline group and $15.2 \%$ and $12.6 \%$ in the Bupropion group compared to $9.0 \%$ and $7.3 \%$ of participants in the placebo group [20-22]. Gonzales et al., [20] reported two serious adverse events attributed to the study treatment: an episode of atrial fibrillation was attributed to Varenicline and a grand mal seizure attributed to Buproprion. Jorenby et al., [21] also reported serious adverse effects including vertigo, hypertension and chest pain in a participant receiving Varenicline and angioedema in a Bupropion recipient.

\section{Nicotine Replacement Therapy (NRT)}

NRT assists in smoking cessation by providing a constant level of nicotine to prevent withdrawal effects [23] and has been reported to be safe and effective in relieving withdrawal symptoms associated with smoking cessation 24,25]. Delivery modes for NRT include Transdermal nicotine (TN), gum, sublingual tablets and oral inhalers [16]. In a meta-analysis of randomised trials, Silagy et al., [26] found no significant difference in cessation rates for different delivery modes, tapering of dose versus abrupt withdrawal and 12 week compared to 28 week treatment [26]. Slowed delivery (i.e. sustained release) of nicotine using TN is considered the preferred form of NRT, as it has the lowest potential for addiction [27] TN is also considered to be the easiest form to use and has the lowest level of adverse effects $[28,29]$.

Hays et al., [29] conducted a double-blind placebo controlled trial which compared the efficacy of six week treatment of daily $22 \mathrm{mg}$ nicotine patches (TN) compared to placebo. They also included an open label arm to compare the efficacy of the same therapy with participants purchasing their own patches. Smoking cessation rates were greater for $\mathrm{TN}(16.8 \% ; 8.7 \%)$ in comparison to placebo $(9.6 \% ; 4.3 \%)$ in the controlled trial at weeks 6 and 24. There was a slight increase in cessation rates in the open label arm of the trial $(19.0 \% ; 10.8 \%)$ at weeks 6 and 24 [29].

Adverse effects of NRT may be associated with delivery mode or the effects of the nicotine itself. Adverse effects that are specific to the mode of delivery include: nasal irritation and runny nose for nasal spray; coughing, throat irritation and oral burning for inhalation; skin rash for TN; jaw pain and hiccups from chewing the gum; dry lips, mouth ulcers and sore throat from sublingual tablets [30]. Nicotine related adverse effects associated with all modes of delivery include, dry mouth, nausea, headache and palpitations [16]. These side effects greatly contribute to the relatively low effect size of these delivery systems in smoking cessation trials.

Cochrane reviews have indicated that the effectiveness of all three treatments are relatively poor with a high degree of relapse at 6 months $[17,31,32]$. Clearly alternative treatments with lower side effects are urgently required for nicotine addiction. For some individuals a new treatment may be important after failing current treatments and therefore additional choice may be important. Additionally a new treatment may also be important if it is more cost effective than current treatments.

\section{Hypericum perforatum and smoking cessation}

The pharmacological properties of Hypericum perforatum (HP) suggest a potential role in reducing addiction to nicotine. HP is the only antidepressant capable of inhibiting the reuptake of the monoamines: serotonin, noradrenaline and dopamine with similar potencies $[33,34]$. HP has previously been found to normalize dopaminergic as well as noradrenergic transmission in the medial PFC $[35,36]$. Recently Ruedeberg et al., [37] have argued for the use of HP in smoking cessation based on dopamine re-uptake in rat striatal cortex after HP administration. HP also inhibits the reuptake of amino acids and neurotransmitters GABA and glutamate $[33,34]$ suggesting that HP both directly and indirectly influences the dopamine reward pathways.

Preclinical research has provided evidence to suggest that chronic HP supplementation also enhances serotoninergic transmission in the PFC [38] and may be of benefit in counteracting the deleterious neural effects of chronic stress [39]. These findings are particularly relevant to individuals undergoing nicotine withdrawal, as smoking abstinence has been found to be associated with elevations in stress hormones [40]. With proven efficacy as an antidepressant for mild to moderate clinical depression [41,42], HP may also assist in cessation of smoking via reduction in depressive symptoms associated with nicotine withdrawal.

Although conventional anti-depressants have been shown to reduce the urges associated with nicotine withdrawal they have not been shown to be efficacious in abstinence [23]. Dopaminergic acting drugs however have been shown to decrease both the symptoms of nicotine withdrawal and abstinence rates but show significant side-effects. As Parsons et al., [43] have argued, because HP acts uniquely on both of these neurotransmitter systems it is important to directly test whether HP directly improves smoking abstinence. Certainly the side-effect profile of HP is significantly better than other treatments for smoking cessation. HP does not appear to have the anticholinergic effects (delirium, sedation, cognitive impairment) of other antidepressants [44] and the adverse effect profile of HP is extremely good compared to other antidepressants [41,42].

The potential benefit of HP on smoking cessation has been investigated in animal studies as well as preliminary clinical trials in 
humans. Barnes et al., [45] conducted an open, uncontrolled pilot study to examine the effects of HP (LI-160) on smoking cessation. Twentyeight participants were administered 300mgor 600mg HP (equivalent to $900 \mathrm{mcg}$ or $1800 \mathrm{mcg}$ hypericin) daily, with treatment commencing one week prior to the quit date and continuing for 12 weeks after quit date. The abstinence rates at 3 months and 12 months after the quit date were $18 \%$ and $0 \%$ respectively for the combined HP treatment groups. Barnes et al., [45] concluded that the results did not provide convincing evidence for the efficacy of HP as an aid in smoking cessation. However there were a number of problems with this trial including the very small sample size, and a run-in period for HP that was too brief for the treatment to be effective. As with most antidepressants, four to six weeks of treatment is typically required to achieve therapeutic benefit [46].

Lawvere et al., [47] also examined the efficacy of a daily dose of $900 \mathrm{mg}$ HP (equivalent to hypericin $2700 \mathrm{mcg}$ ) on cessation rates of 24 smokers in an open-label trial. Treatment commenced a week before quit date and continued a further 12 weeks after quit date. Thirty-five percent of participants were found to be smoke free at 12 weeks after the quit date. Anxiety was also found to be significantly reduced from the third to the twelfth week after the quit date, and a decrease in depression scores and nicotine withdrawal symptoms was evident during the 12 week course of the study [47]. Lawvere et al., [47] concluded that HP may prove to be a useful adjunctive therapy for smoking cessation.

In a more recent placebo controlled clinical trial Parsons et al., [43] examined the efficacy of $900 \mathrm{mg}$ HP daily (equivalent to hypericin $1080-2520 \mathrm{mcg}$ ) versus placebo for 16 weeks. In a factorial design participants were also allocated chromium or placebo. There were no significant differences between the SJW and the placebo group at either 4 or 16 weeks indicating that the HP used in this study was not effective in smoking abstinence. Surprisingly there was also no significant difference in any of the mood related variables or urges to smoke between the HP and placebo groups indicating that the HP in this trial did not effectively increase serotononergic or dopaminergic activity which would have decreased nicotine cravings and negative moods. In a similar trial using another different HP extract, Sood et al., [48] administered $900 \mathrm{mg} \mathrm{HP}$ /day versus $1800 \mathrm{mg} \mathrm{HP} /$ day (equivalent to hypericin $2700 \mathrm{mcg}$ and hypericin $5400 \mathrm{mcg}$ ) for 12 weeks in 118 participants. Sood et al., [48] reported that there were no significant differences in abstinence rates between either of the HP groups or placebo at both 12 or 24 weeks post-treatment.

In consideration of the strong preclinical evidence of central dopaminergic effects associated with HP $[35,36]$, the mixed results of these human trials are hard to reconcile. One possible explanation for the different findings for each of these trials may be attributed to use of different HP extracts, with differences in quality across different manufacturers. Unlike pharmaceuticals in which an active compound is concentrated and reliably reproduced from batch to batch, a nutraceutical like HP may significantly differ for a number of factors including climatic conditions during growth, geographical location, extraction technique, as well as handling and transportation techniques.

\section{Ze117 standardized Hypericum perforatum extract}

Ze $117\left(\right.$ Remotiv $\left.^{\circledR}\right)$ is a HP extract which has been widely tested for its safety and efficacy and has been clinically proven to be as effective as, and better tolerated than antidepressants in relieving depressive symptoms [49-51]. Ze 117 is a standardised product containing 1.375 g HP herb, 500 mcg hypericin and negligible levels of hyperforin [52] which is the constituent believed responsible for interactions with other medications [53,54]. Ze 117 has been used in an estimated 6 million patients in the last 10 years, with an extremely low incidence of reported side effects in trials and in clinical use (Periodic Safety Update Report, Ze 117 March 2004).

A pilot study from our laboratory conducted in 2006 examined the effects of Ze117 HP extract (Ze117; 500mcg Hypericin 2/d) in the management of smoking cessation compared to a transdermal nicotine patch (Nicabate CQ; step down regime commencing at $24 \mathrm{mg} /$ day) [55] Forty Participants aged between 18 and 60 years who had smoked for at least 5 years and were wishing to quit smoking were enrolled in the study. The study was conducted over a 14 and 10 week administration period for the HP and NRT groups, respectively. The results of the study revealed that participants in both the HP $(n=17)$ and NRT $(n=12)$ groups decreased the number of cigarettes smoked per day over the 12 week treatment period. Quit rates for the HP and TN groups were not statistically different indicating that there were equivalent numbers of smokers from both the HP and TN groups who were able to quit smoking.

\section{The current study}

The current study extended on the methodology of the initial pilot study from our laboratory, examining the efficacy of Hypericum perforatum (HP; ZE117) in the management of smoking cessation, both as monotherapy and as augmentation to Transdermal NRT for smoking cessation in a larger sample. Our outcome measure was abstinence rate (primary outcome variables) determined by $\mathrm{CO}$ measurement. Secondary outcome measures included the number of cigarettes smoked, cravings and withdrawal symptoms associated with smoking cessation. Given the similar pharmacological profile to Buproprion, it was hypothesized that there would be no difference in our primary outcome variable between the Ze117 or NRT groups. It was also hypothesized that combined Ze117/NRT would work more effectively than either NRT or Ze117 used in isolation.

\section{Clinical significance}

The results may have significant benefits for influencing health promotion policies in Australia (and internationally) and may help encourage current smokers to attempt to quit either for the first time or after one or more failed attempts. If proven effective the cost of Ze117 is also highly advantageous in relative terms to other current treatments. Current treatments are only at best moderately effective. According to the American Cancer Society (Nov 2013) between 4 and $7 \%$ of smokers are able to quit smoking after any attempt and only about $25 \%$ of smokers are able to successfully quit smoking with the help of current pharmaceutical interventions. Smokers who fail to quit smoking show significant negative effects on their quality of life, their health and the community by way of economic burden of caring for patients with cancer, coronary heart disease and other diseases due to tobacco smoking. An additional effective treatment will add to our current treatment measures.

\section{Methods}

\section{Participants}

Sixty volunteers, aged between 18 and 60 years, were recruited to participate in the trial. All interested individuals were screened over the phone by the research officer to assess their suitability for participation in the study based on the following selection criteria: Smoking for at least 5 years and currently smoking at least 10 cigarettes per day, at least one prior attempt at quitting smoking, motivation to quit 
smoking, weighing more than $45 \mathrm{~kg}$, no history of anxiety/depression, not currently taking any prescription medications, no other chronic health conditions, not currently or in past 6 months taking HP or antidepressants, not currently or in past 6 months classified as being addicted to any substances apart from nicotine, not currently using NRT and not pregnant or lactating. Once deemed suitable to participate in the study participants committed to a quit date with the research officer and were booked in for their first testing session 4 weeks prior to their quit date. All testing was conducted at Swinburne University Hawthorn Campus. All participants provided informed consent to participate in the study which was approved by Swinburne University Human Research Ethics committee.

\section{Study design}

The study was conducted over a 14 week period at the Brain Sciences Institute at Swinburne University of Technology, Hawthorn. Following the first (baseline) visit, participants were randomly allocated to one of three treatment groups: Group 1-Hypericum perforatum (Ze117; 500 mcg $\times 2$ daily), Group 2-Transdermal NRT (Nicabate CQ clear) and Group 3-Hypericum perforatum (Ze117; $500 \mathrm{mcg} \times 2$ daily) and Transdermal NRT (Nicabate CQ clear).

The primary endpoints were: (1) The mean number of smokers in each treatment group, as defined by a reading of $\geq 6 \mathrm{ppm}$ on the Benfont Smokerlyzer at Visit 4, 10 weeks post quitting smoking. (2) Change in the mean number of cigarettes smoked per week in each treatment group at Visit 4, 10 weeks post quitting smoking in comparison to Baseline. The secondary endpoints were state anxiety measured by the State Trait Anxiety Inventory (STAI), DSM criteria for nicotine dependence \& Fagerstrom dependence measures.

\section{Investigational treatments}

Remotiv $^{\circledR}$ Ze117: Ze117 is a high quality standardized HP extract manufactured by Zeller (Switzerland) which contains $1.375 \mathrm{~g}$ HP herb and $500 \mathrm{mcg}$ hypericin. Ze117 has clinically established safety and efficacy as an antidepressant [49-51]. As with most antidepressants, it takes four to six weeks administration of Ze117 to achieve therapeutic benefits [46]. Therefore, participants taking Ze117 commenced a washin period $(500 \mathrm{mcg}$ hypericin $\times 2 / \mathrm{d}$ ) of four weeks ( 28 days) prior to the quit date. Ze117 is sold in Australia by Flordis under the registered trademark Remotiv ${ }^{\mathbb{R}}$.

Nicabate CQ (clear): It is an over the counter pharmaceutical and treatment consists of a ten week step down process consisting of three stages: Step 1: Weeks 1-5-Six weeks on a patch delivery of $21 \mathrm{mg}$ nicotine over 24 hours; Step 2: Weeks 6-7-Two weeks on a patch delivering 14 $\mathrm{mg}$ of nicotine over 24 hours; Step 3: Weeks 8-10-Two weeks on a $7 \mathrm{mg}$ of nicotine delivery over 24 hours.

\section{Materials}

Smoking abstinence was confirmed using The Bedfont Compact Smokerlyser (Bedfont instruments, Kent, UK). The Bedfont Smokerlyser measures carbon monoxide (CO) levels in the breath in parts per million (ppm). This measurement is based on the conversion of $\mathrm{CO}$ to $\mathrm{CO}_{2}$ over a catalytically active electrode. When the breath is held, equilibrium is established between $\mathrm{CO}$ in the blood and $\mathrm{CO}$ in alveolar air, therefore there is a high degree of correlation between breath $\mathrm{CO}$ levels and $\mathrm{COHb}$ concentration [56]. In the current study, a measurement of 6 ppm was taken as confirmation of the smoking status of participants. This is a more stringent cut-off value used by Parsons et al., [43] who used $10 \mathrm{ppm}$ and Sood et al., [48] who used 8 ppm.
The Fagerström Test for Nicotine Dependence (FTND) [57] is an 8 -item self-report questionnaire, with a total score $\geq 7$ indicative of high nicotine dependence. The State-Trait Anxiety Inventory (STAI) [58] is a self-report assessment device consisting of two parts: (i) State Anxiety, a 20 -item scale measuring an individual's transitory feelings of tension and apprehension, and (ii) Trait Anxiety, a 20-item scale measuring relatively stable individual differences in anxiety proneness. In this paper we report the state component of the STAI.

\section{Procedure}

Participants were required to attend Swinburne University for an initial baseline visit 4 weeks prior to quitting cigarettes, then again for a retest at week 4 (quit date), week 10 (6 weeks post quit) and week 14 (10 weeks post quit).

Visit 1: Baseline visit Pre-quit (Week 0): Participants visited Swinburne University 4 weeks prior to their quit date for the first testing session. During this first visit, participants completed a general questionnaire regarding their health, demographics, vitals (blood pressure, pulse rate), educational and social economic status, and smoking habits. During each testing session the following assessments were administered: the Fagerström Test for Nicotine dependence (FTND) as a measure of smoking dependence. The State-Trait Anxiety Inventory (STAI), a self-report assessment device designed to measure participants' subjective feelings of tension and apprehension. Carbon monoxide breath analysis performed using the Bedfont Micro Smokerlyser to verify participant smoking status, with readings above $6 \mathrm{ppm}$ confirming that the participant is a smoker. DSM IV structured questionnaire, to measure Nicotine withdrawal symptoms and cravings.

A participant self-report diary was used to monitor adverse events and the number of cigarettes smoked during the quit period, with quitting rate expressed as the percentage of participants with confirmed abstinence. To help increase compliance, participants were asked to email or post the self-report diary to Swinburne University on a weekly basis. During the first 4 weeks of the trial (4 weeks pre-quit date) participants randomized to the HP group were required to start taking the recommended HP dose, for 28 days prior to the quitting smoking.

Visit 2: Quit date (Week 4). On the quit date all participants were asked to continue taking these treatments for the remaining treatment duration period. Participants in the NRT treatment groups (groups 2\&3) commenced treatment with $21 \mathrm{mg}$ dose patches for 6 weeks.

Visit 3: 6 weeks post quit date (Week 10): On the third visit participants in the NRT group ceased taking the $21 \mathrm{mg}$ treatment dose and commenced the $14 \mathrm{mg}$ patch dosage for 2 weeks, followed by the final step down process of $7 \mathrm{mg}$ patches per day for the final 2 weeks of the treatment period. The trial was then continued for a further 4 weeks with a fourth testing session conducted at week 14 (10 weeks post quit date) of the trial using the same tests.

Visit 4: 10 weeks post-quit (Week 14). Participants from all three groups discontinued treatment and visited the BSI for follow up testing.

\section{Statistical analysis}

For the primary outcome variables non-parametric tests were used to assess differences in the number of smokers at week 14 identified by the Bedfont $\mathrm{CO}$ measure. For the secondary measures, repeated measures parametric analysis of variance was used to examine both changes from week 4 to week 14 (Time) and differences in changes in the measures from week to 14 that may differ between the groups (Time by Treatment). 
Citation: Stough C, Scholey A, Kure C, Tarasuik J, Kras M, et al. (2013) An Open Label Study investigating the Efficacy of Hypericum perforatum Special Extract (ZE117), Nicotine Patches and Combination (ZE117)/Nicotine Patches for Smoking Cessation. Altern Integ Med 2: 147. doi:10.4172/2327-5162.1000147

Page 5 of 7

\section{Results}

\section{Primary Outcome: Smoking status at week 14}

Analysis of the number of current smokers as assessed by the Bedfont Smokerlyzer (6 ppm) was used as the primary outcome variable. A score of 1 denotes negative test (i.e. non-smoker with a Bedfont score of less than $6 \mathrm{ppm}$ ) and a score of 2 denotes a positive $\mathrm{CO}$ test (i.e. smoker with a Bedfont score of 6 or greater) (Table 1).

There was no statistical difference between the three groups in the number of smokers at either the quit date (week 4) or at the completion of the study (Week 14, 10 post-quit). For the assessment of smoking status at week 4 all participants were smokers according to the Bedfont $\mathrm{CO}$ values. Using non-parametric statistics to assess differences in the number of smokers at the end of the treatment date-week 14 (10 weeks after the quit date) no statistically significant differences between the groups were found (Kruskal Wallis Independent samples test $=.483$ $\mathrm{df}=2, \mathrm{p}>.785)$.

\section{Secondary outcome variables}

We also examined data for the STAI (state anxiety), DSM criteria \& Fagerström dependence measures for both the factors of time (changes in scores between the quit date and week 14 (completion of the treatment) as well as time by treatment factor (changes in time between the three groups). The mean and SDs for these variables are presented in Table 2.

Fagerström Tolerance was found to be significantly reduced at 10 weeks following the quit date across treatment groups $\mathrm{F}=22.7$, $\mathrm{P}<.001$, although the treatment $\times$ time interaction was found to be non-significant $\mathrm{F}=1.8 \mathrm{NS}$. Fagerström Dependence was found to be significantly increased at 10 weeks following the quit date $\mathrm{F}=12.8$, $\mathrm{P}<.001$, with the treatment $\times$ time interaction also non-significant $\mathrm{F}=.72$ NS. Fagerström Craving was found to be significantly reduced at 10 weeks following the quit date across treatment groups $\mathrm{F}=25.9, \mathrm{P}<.001$, and the treatment $\times$ time interaction was also found to be significant $\mathrm{F}=5.5, \mathrm{p}<.01$. Fagerström Relapse (positive) was found to be significantly increased at 10 weeks following the quit date $\mathrm{F}=19.3, \mathrm{P}<.001$, with the treatment $\times$ time interaction non-significant $\mathrm{F}=1.4$ NS. Fagerström Relapse (negative) was found to be significantly increased at 10 weeks following the quit date $\mathrm{F}=32.0, \mathrm{P}<.001$, with the treatment $\times$ time interaction non-significant $\mathrm{F}=2.3 \mathrm{NS}$. State Anxiety was now found to be significantly changed at 10 weeks following the quit date $\mathrm{F}=.38$, NS, and neither was the treatment $\times$ time interaction significant $\mathrm{F}=.86 \mathrm{NS}$.

\section{Discussion}

The finding of no significant differences between groups in the numbers of smokers at 10 weeks following the quit date indicates that each treatment condition was equally effective in smoking cessation as measured by $\mathrm{CO}$ criteria of $6 \mathrm{ppm}$. Interestingly there is a small trend that is suggestive of the combined treatment condition working best but this would only reach statistical significance in a much larger trial.

\begin{tabular}{|l|c|c|}
\hline & Visit 2 Quit date & Visit 4 10 weeks post quit \\
\hline Ze117 group & (SDs) & (SDs) \\
\hline $\begin{array}{l}\text { Nicotine patches (NRT) } \\
\text { group }\end{array}$ & $1.27(.46)$ & $1.60(.50)$ \\
\hline Ze117/NRT group & $1.18(.39)$ & $1.60(.51)$ \\
\hline
\end{tabular}

Table 1: Mean number of participants classified as smokers at quite date and 10 weeks after treatment based on $\mathrm{CO}$ value of $6 \mathrm{ppm}(1=$ non-smoker-less than 6 ppm; $2=$ smoker 6 ppm or greater).

\begin{tabular}{|l|l|c|c|c|c|}
\hline \multirow{3}{*}{ Variable } & Group & \multicolumn{2}{|c|}{$\begin{array}{c}\text { Week 4 } \\
\text { (quit date) }\end{array}$} & \multicolumn{2}{c|}{ Week 14 } \\
(10 weeks post quit)
\end{tabular}

Table 2: Means, SDs for secondary outcome variables at week 4 (quite date) and week 14 (10 weeks of treatment).

Additionally future research should also use urinary measures to assess abstinence. Compared to urinary cotinine, $\mathrm{CO}$ has a shorter half-life and therefore the rate of false negative classifications using $\mathrm{CO}$ measurements may be higher. This will have implications for participation rates as well as costs in future studies but urinary measures provide the strongest evidence of abstinence. Nevertheless, CO measurements have been used previously in all of the HP smoking cessation studies and provide an efficient and consistent measure in which to interpret the findings of the current study within the background of previous studies.

Given the equivalence of our primary measure between treatment groups, an analysis of the secondary measures was helpful in revealing potential differences between treatments in the context of smoking cessation.There was a significant time interaction for all variables except state anxiety. This suggests that across all three interventions (HP, NRT and combined HP/NRT) there were significant changes in our secondary measures except for state anxiety. These changes were in the expected direction based on previous studies indicating that feelings of dependence generally increased and tolerance decreased over the course of the intervention. In terms of the differential effects of the treatments, there were no significant differences in the time by treatment effects for all of our secondary variables except for craving. For this variable the HP treatment was best at reducing feelings of craving and this is consistent with the known pharmacological action of $\mathrm{HP}$ as well as previous research which has shown reductions in craving after HP administration [42]. Reducing craving may be an important factor in smoking cessation for many smokers and this mechanism may help explain the equivalence in cessation across the three different treatments arms observed in the present study. Clearly there are other mechanisms independent of craving that can be attributable to the efficacy of NRT observed in the current study.

There were some important components to the present trial. First, this was the first trial to examine this particular HP extract (Ze117) on smoking cessation. Ze117 is a high quality well-researched HP extract that has proven consistency, potency and quality. Another important component to this trial was that it included for the first time a combined HP/NRT arm. Given the different pharmacological actions of HP and NRT it could have been reasonably expected that the 
Citation: Stough C, Scholey A, Kure C, Tarasuik J, Kras M, et al. (2013) An Open Label Study investigating the Efficacy of Hypericum perforatum Special Extract (ZE117), Nicotine Patches and Combination (ZE117)/Nicotine Patches for Smoking Cessation. Altern Integ Med 2: 147. doi:10.4172/2327-5162.1000147

Page 6 of 7

combination treatment would show greater effect in terms of smoking cessation than either intervention alone. This was not the case. In fact the only significantly different effect observed in the current study was the significant decrease in symptoms of craving observed in the Ze117 treatment arm.

The purpose of this study was to examine whether there was preliminary evidence for Ze117 rather than HP in general as a treatment option for smoking cessation. To adequately examine the efficacy of HP as a treatment option for smoking cessation a study in which several different HP extracts would need to be compared for smoking cessation. Therefore the results of this study can only generalise in terms of the specific HP extract used in the current study. As such there is now preliminary evidence justifying a considerably larger randomized double blind study administering this particular HP extract (Ze117) for smoking cessation. Data from this large study if confirming the pilot data could lead to the use of Ze117 for smoking cessation in addition to NRT.

The smoking cessation market is currently a multi-billion dollar industry and many smokers fail to give up smoking after NRT treatment. Additionally NRT and other treatments show a considerably higher side-effect profile than HP in general and Ze117 specifically. Increasing choice of treatments may have important consequences for reducing the burden of smoking related diseases in many countries. Future studies should also employ more mechanistic measures and examine interactions between nicotine and Ze117 for cytochrome enzymes (i.e. safety data). Given the significant health effects of smoking, alternative treatments for smoking cessation are urgently required. The results of this study, although preliminary provide a strong framework for larger efficacy and safety studies.

\section{References}

1. Ingersoll KS, Cohen J (2005) Combination treatment for nicotine dependence: state of the science. Subst Use Misuse 40: 1923-1943, 2043-2048.

2. Ridolfo B, Stevenson C (2001) The quantification of drug-caused morbidity and treatment in Australia. 1998, Australian Institute of Health and Welfare Canberra.

3. Collins DJ, Lapsley HM (2002) Counting the costs: Estimates of the social costs of drug abuse in Australia in 1998-9. Monograph No 49. National Drug Strategy 2002.

4. Zhu S, Melcer T, Sun J, Rosbrook B, Pierce JP (2000) Smoking cessation with and without assistance: a population-based analysis. Am J Prev Med 18: 305311.

5. Pierce RC, Kumaresan $V(2006)$ The mesolimbic dopamine system: the final common pathway for the reinforcing effect of drugs of abuse? Neurosci Biobehav Rev 30: 215-238.

6. Heimer L, Alheid GF, de Olmos JS, Groenewegen HJ, Haber SN, et al. (1997) The accumbens: beyond the core-shell dichotomy. J Neuropsychiatry Clin Neurosci 9: 354-381.

7. Tomkins DM, Sellers EM (2001) Addiction and the brain: the role of neurotransmitters in the cause and treatment of drug dependence. CMAJ 164: 817-821.

8. Klink R, de Kerchove d'Exaerde A, Zoli M, Changeux JP (2001) Molecular and physiological diversity of nicotinic acetylcholine receptors in the midbrain dopaminergic nuclei. J Neurosci 21: 1452-1463.

9. Balfour DJ (2004) The neurobiology of tobacco dependence: a preclinical perspective on the role of the dopamine projections to the nucleus accumbens [corrected]. Nicotine Tob Res 6: 899-912.

10. Di Chiara G (2000) Role of dopamine in the behavioural actions of nicotine related to addiction. Eur J Pharmacol 393: 295-314.

11. Seth P, Cheeta S, Tucci S, File SE (2002) Nicotinic--serotonergic interactions in brain and behaviour. Pharmacol Biochem Behav 71: 795-805.
12. Foulds $J$ (1999) The psychology of tobacco addiction: why it is difficult to stop smoking. Monaldi Arch Chest Dis 54: 495-497.

13. Awtry TL, Frank JG, Werling LL (2006) In vitro regulation of serotonin transporter activity by protein kinase $\mathrm{A}$ and nicotinic acetylcholine receptors in the prefrontal cortex of rats. Synapse 59: 342-349.

14. Awtry TL, Werling LL (2003) Acute and chronic effects of nicotine on serotonin uptake in prefrontal cortex and hippocampus of rats. Synapse 50: 206-211.

15. Rasmussen K, Czachura JF (1997) Nicotine withdrawal leads to increased sensitivity of serotonergic neurons to the $5-\mathrm{HT} 1 \mathrm{~A}$ agonist $8-\mathrm{OH}-\mathrm{DPAT}$ Psychopharmacology (Berl) 133: 343-346.

16. MIMS Australia (2006) MIMS annual: St Leonards, N.S.W,CMP Medica Australia

17. Stead LF, Perera R, Bullen C, Mant D, Hartmann-Boyce J (2008) Nicotine replacement therapy for smoking cessation. Cochrane Database Syst Rev.

18. Schnoll RA, Lerman C (2006) Current and emerging pharmacotherapies for treating tobacco dependence. Expert Opin Emerg Drugs 11: 429-444.

19. Hurt RD, Sachs DP, Glover ED, Offord KP, Johnston JA, et al. (1997) A comparison of sustained-release bupropion and placebo for smoking cessation N Engl J Med 337: 1195-1202.

20. Gonzales D, Rennard SI, Nides M, Oncken C, Azoulay S, et al. (2006) Varenicline, an alpha4beta2 nicotinic acetylcholine receptor partial agonist, vs sustained-release bupropion and placebo for smoking cessation: a randomized controlled trial. JAMA 296: 47-55.

21. Jorenby DE, Leischow SJ, Nides MA, Rennard SI, Johnston JA, et al. (1999) A controlled trial of sustained-release bupropion, a nicotine patch, or both for smoking cessation. N Engl J Med 340: 685-691.

22. Keating GM, Siddiqui MA (2006) Varenicline: a review of its use as an aid to smoking cessation therapy. CNS Drugs 20: 945-960.

23. Peters MJ, Morgan LC (2002) The pharmacotherapy of smoking cessation Med J Aust 176: 486-490.

24. Fiore MC, Kenford SL, Jorenby DE, Wetter DW, Smith SS, et al. (1994) Two studies of the clinical effectiveness of the nicotine patch with different counseling treatments. Chest 105: 524-533.

25. Lavelle C, Birek C, Scott DA (2003) Are nicotine replacement strategies to facilitate smoking cessation safe? J Can Dent Assoc 69: 592-597.

26. Silagy C, Lancaster T, Stead L, Mant D, Fowler G (2004) Nicotine replacement therapy for smoking cessation. Cochrane Database Syst Rev.

27. Hajek P, McRobbie H, Gillison F (2007) Dependence potential of nicotine replacement treatments: effects of product type, patient characteristics, and cost to user. Prev Med 44: 230-234.

28. Fiore MC (2000) Treating tobacco use and dependence: an introduction to the US Public Health Service Clinical Practice Guideline. Respir Care 45: 11961199 .

29. Hays JT, Croghan IT, Schroeder DR, Offord KP, Hurt RD, et al. (1999) Over-thecounter nicotine patch therapy for smoking cessation: results from randomized, double-blind, placebo-controlled, and open label trials. Am J Public Health 89: 1701-1707.

30. Schneider NG, Olmstead R, Nilsson F, Mody FV, Franzon M, et al. (1996) Efficacy of a nicotine inhaler in smoking cessation: a double-blind, placebocontrolled trial. Addiction 91: 1293-1306.

31. Cahill K, Stead LF, Lancaster T (2008) Nicotine receptor partial agonists for smoking cessation. Cochrane Database Syst Rev.

32. Hughes JR, Stead LF, Lancaster T (2007) Antidepressants for smoking cessation. Cochrane Database Syst Rev.

33. Nathan $P$ (1999) The experimental and clinical pharmacology of St John's Wort (Hypericum perforatum L.) Mol Psychiatry 4: 333-338.

34. Nathan PJ (2001) Hypericum perforatum (St John's Wort): a non-selective reuptake inhibitor? A review of the recent advances in its pharmacology. $J$ Psychopharmacol 15: 47-54

35. Butterweck V, Böckers T, Korte B, Wittkowski W, Winterhoff H (2002) Longterm effects of St. John's wort and hypericin on monoamine levels in rat hypothalamus and hippocampus. Brain Res 930: 21-29. 
Citation: Stough C, Scholey A, Kure C, Tarasuik J, Kras M, et al. (2013) An Open Label Study investigating the Efficacy of Hypericum perforatum Special Extract (ZE117), Nicotine Patches and Combination (ZE117)/Nicotine Patches for Smoking Cessation. Altern Integ Med 2: 147. doi:10.4172/2327-5162.1000147

Page 7 of 7

36. Yoshitake T, lizuka R, Yoshitake S, Weikop P, Müller WE, et al. (2004) Hypericum perforatum $L$ (St John's wort) preferentially increases extracellular dopamine levels in the rat prefrontal cortex. Br J Pharmacol 142: 414-418.

37. Ruedeberg C, Wiesmann UN, Brattstroem A, Honegger UE (2010) Hypericum perforatum L. (St John's wort) extract Ze 117 inhibits dopamine re-uptake in rat striatal brain slices. An implication for use in smoking cessation treatment? Phytother Res 24: 249-251.

38. Mannucci C, Pieratti A, Firenzuoli F, Caputi AP, Calapai G (2007) Serotonin mediates beneficial effects of Hypericum perforatum on nicotine withdrawal signs. Phytomedicine 14: 645-651.

39. Trofimiuk E, Braszko JJ (2008) Alleviation by Hypericum perforatum of the stress-induced impairment of spatial working memory in rats. Naunyn Schmiedebergs Arch Pharmacol 376: 463-471.

40. Pickworth WB, Fant RV (1998) Endocrine effects of nicotine administration, tobacco and other drug withdrawal in humans. Psychoneuroendocrinology 23 : 131-141.

41. Kim HL, Streltzer J, Goebert D (1999) St. John's wort for depression: a metaanalysis of well-defined clinical trials. J Nerv Ment Dis 187: 532-538.

42. Linde K, Ramirez G, Mulrow CD, Pauls A, Weidenhammer W, et al. (1996) St John's wort for depression--an overview and meta-analysis of randomised clinical trials. BMJ 313: 253-258.

43. Parsons A, Ingram J, Inglis J, Aveyard P, Johnstone E, et al. (2009) A proof of concept randomised placebo controlled factorial trial to examine the efficacy of St John's wort for smoking cessation and chromium to prevent weight gain on smoking cessation. Drug Alcohol Depend 102: 116-122.

44. Khalifa AE (2001) Hypericum perforatum as a nootropic drug: enhancement of retrieval memory of a passive avoidance conditioning paradigm in mice. $J$ Ethnopharmacol 76: 49-57.

45. Barnes J, Barber N, Wheatley D, Williamson EM (2006) A pilot randomised, open, uncontrolled, clinical study of two dosages of St John's wort (Hypericum perforatum) herb extract (LI-160) as an aid to motivational/behavioural support in smoking cessation. Planta Med 72: 378-382.

46. Bennett DA Jr, Phun L, Polk JF, Voglino SA, Zlotnik V, et al. (1998) Neuropharmacology of St. John's Wort (Hypericum). Ann Pharmacother 32: 1201-1208.
47. Lawvere S, Mahoney MC, Cummings KM, Kepner JL, Hyland A, et al. (2006) A Phase II study of St. John's Wort for smoking cessation. Complement Ther Med 14: 175-184.

48. Sood A, Ebbert JO, Prasad K, Croghan IT, Bauer B, et al. (2010) A randomized clinical trial of St. John's wort for smoking cessation. J Altern Complement Med 16: 761-767

49. Friede M, Henneicke von Zepelin HH, Freudenstein J (2001) Differential therapy of mild to moderate depressive episodes (ICD-10 F 32.0; F 32.1) with St. John's wort. Pharmacopsychiatry 34 Suppl 1: S38-41.

50. Schrader E (2000) Equivalence of St John's wort extract (Ze 117) and fluoxetine: a randomized, controlled study in mild-moderate depression. Int Clin Psychopharmacol 15: 61-68.

51. Woelk H (2000) Comparison of St John's wort and imipramine for treating depression: randomised controlled trial. BMJ 321: 536-539.

52. Wurglics M, Westerhoff K, Kaunzinger A, Wilke A, Baumeister A, et al (2001) Batch-to-batch reproducibility of St. John's wort preparations Pharmacopsychiatry 34 Suppl 1: S152-156.

53. Hall SD, Wang Z, Huang SM, Hamman MA, Vasavada N, et al. (2003) The interaction between St John's wort and an oral contraceptive. Clin Pharmacol Ther 74: 525-535.

54. Obach RS (2000) Inhibition of human cytochrome P450 enzymes by constituents of St. John's Wort, an herbal preparation used in the treatment of depression. J Pharmacol Exp Ther 294: 88-95.

55. Stough C, Kure C, Tarasuik J, Lloyd J (2006) A clinical trial comparing St John's Wort (Hypericum perforatum) and Nicotine replacement in the Management of Smoking Cessation Unpublished Clinical Trial Brain Sciences Institute, Swinburne University of Technology.

56. Middleton ET, Morice AH (2000) Breath carbon monoxide as an indication of smoking habit. Chest 117: 758-763.

57. Heatherton TF, Kozlowski LT, Frecker RC, Fagerström KO (1991) The Fagerström Test for Nicotine Dependence: a revision of the Fagerström Tolerance Questionnaire. Br J Addict 86: 1119-1127.

58. Spielberger CD, Goruch RL, Lushene PR, Vagg PR, Jacobs AG (1983) Manual for the State-Trait Anxiety Inventory (Form Y). Palo-Alto: Consulting Psychologists Press, Inc. 